\title{
MODELADO DE LA CALIDAD DEL AIRE URBANO. UN EXPERIMENTO METODOLÓGICO CON TÉCNICAS DE INTERPOLACIÓN ESPACIAL
}

\author{
María Rosa Cañada Torrecilla, Antonio Moreno Jiménez y Heizel González Lorenzo \\ Departamento de Geografía. Universidad Autónoma de Madrid \\ rosa.canada@uam.es, antonio.moreno@uam.es, heizelglez@gmail.com
}

\section{RESUMEN}

La obtención de estimaciones de los niveles de polución para todo el espacio de una ciudad es una necesidad básica que adolece de dificultades serias por las deficiencias en los datos muestrales registrados en las estaciones de medición. El objetivo de este trabajo estriba en establecer un conjunto de decisiones técnicas para mejorar dichas estimaciones, empleando modelos e información asequibles. A tal fin se presenta un protocolo procedimental que conlleva, por un lado, la exploración y caracterización del patrón espacial de la polución a partir de los datos muestrales y, por otro, la aplicación de dos métodos de interpolación, uno geoestadístico, el «Kriging», y otro determinista, la media ponderada por el inverso de la distancia (IDW). El uso de criterios estadísticos y contextuales para comparar y valorar los resultados ayuda a elegir una solución razonablemente rigurosa y más consistente con el ámbito local.

Palabras clave: Modelado espacial, interpolación, calidad ambiental, contaminación urbana, sistemas de información geográfica.

\begin{abstract}
Achieving detailed estimates of pollution levels for the whole space of a city is a basic need suffering serious drawbacks due to deficiencies in sampling data provided by monitoring stations. This work aims to establish a set of technical decisions helping to improve such estimates, using available models and affordable data. To this end a procedural protocol is
\end{abstract}

Fecha de recepción: septiembre 2012.

Fecha de aceptación: septiembre 2013. 
presented involving, on the one hand, the exploration and characterization of spatial pollution patterns, and on the other, the applications of two interpolation methods: one geostatistical (Kriging) and another deterministic (Inverse Distance Weighting, IDW). The use of statistical and contextual criteria for comparing and assessing the results aids to select a solution both reasonably rigorous and more locally consistent.

Key words: Spatial modeling, interpolation, environmental quality, urban pollution, geographical information systems.

\section{INTRODUCCIÓN}

La ley de protección del aire y de calidad de la atmósfera (LEY 34/2007) dice en su preámbulo que la atmósfera es un bien común indispensable para la vida y que todas las personas tienen derecho a su uso y disfrute, pero también tienen la obligación de su conservación. Dado que el aire es un recurso vital y que su contaminación puede ocasionar daños para la salud humana y el medio ambiente, la protección y la calidad de la atmósfera son desde hace años metas obligadas de política ambiental; por esta razón el 13 de abril de 2013 fue aprobado por el Consejo de Ministros el Plan Nacional de Calidad del aire y Protección de la Atmósfera 2013-2016 (Plan Aire). A medida que los procesos de industrialización y de urbanización territorial se han ido desarrollando han provocado una degradación de la calidad del aire, convirtiéndose la contaminación atmosférica de las ciudades en un problema de salud pública universal, y en una de las prioridades mundiales más importantes según la OMS.

Uno de los intereses por la polución urbana recae en medir las desigualdades ambientales dentro de la ciudad y sobretodo en relacionarlas con los grupos sociales que las soportan, es decir, importa la contaminación desde la óptica de la justicia ambiental. Este es un tema de investigación preferente internacionalmente, pero que en España está poco abordado aún y que está siendo impulsado por un equipo de la Universidad Autónoma de Madrid, mediante una serie de proyectos de ámbito nacional e internacional.

Es bien patente que el conocimiento de los niveles de polución a nivel local, y singularmente en el interior de las ciudades, resulta insuficiente por las limitaciones derivadas de la cifra y de la distribución espacial de las estaciones de medición. Realmente solo unos pocos lugares (emplazamientos) poseen tales datos, pero generalmente se carece de valores para todo el espacio intersticial, de modo que permitan una apreciación más completa de la calidad del aire. De esta forma los análisis y valoraciones basados en las estaciones de medición exclusivamente, sin perjuicio de su utilidad y relevancia, resultan extremadamente incompletos y con una representatividad limitada. Disponer de una cartografía espacialmente detallada de la contaminación urbana, como base para identificar las zonas expuestas a niveles inaceptables o peligrosos constituye una necesidad perentoria de nuestras sociedades y para la Geografía constituye un frente de contribución relevante, como certeramente argumentó Monmonier (1997). Ello posibilitaría adentrarse en terrenos apenas abordados todavía, como la exposición de la población a la contaminación, la evaluación de los riesgos asociados, la determinación de impactos y la eventual explicación de posibles consecuencias.

Superar esta situación exige instrumentos de estimación, los cuales constituyen en las geociencias un frente de desarrollo científico importante. La estimación de fenómenos como la 
contaminación en el espacio ha recurrido a modelos de muy diversa naturaleza, como vía para aproximarse a la distribución espacial de los mismos y, por extensión, a sus implicaciones.

En escenarios con altos recursos, i. e. con una buena información relativa a los focos de emisión y a los factores de difusión espacial, así como con excelentes tecnologías de cálculo, se han adoptado técnicas de simulación que estiman la dispersión, niveles de inmisión y deposición, incluso a escalas grandes, e. g. para el entorno inmediato de un foco contaminante o para cada una de las vías públicas de la zona. Véanse, por ejemplo, la breve revisión de tales modelos realizada por Daly y Zannetti (2007), los estudios de Baldasano et al. (2008), que desarrollan un modelo de transporte químico de alta resolución para estimar la contaminación en España, o el de Parra (2004) que, utilizando también un modelo de transporte químico, analiza la dispersión del ozono en Cataluña, para lo cual se necesita conocer las emisiones atmosféricas en la zona, los campos de viento y otros parámetros relacionados con la energía turbulenta.

Cuando ello no es posible, una opción menos exigente de información y que hace viable obtener estimaciones (aunque con menos exactitud) estriba en los interpoladores espaciales, los cuales forman una familia muy reconocida y relevante en las geociencias. Con ellos, a partir de una muestra de observaciones empíricas, se avista obtener unas estimaciones de los valores que habría en los lugares carentes de tales mediciones. La adscripción espacial de los datos observados a objetos poligonales, lineales o puntuales ha generado una amplia variedad de lógicas y modelos para abordar ese reto, sobre lo cual existe una bibliografía notable. Nuestra aportación versa concretamente sobre uno de tales tipos de objetos, los puntos, los cuales constituyen una de las formas más habituales de recoger y representar mediciones instrumentales sobre el terreno de atributos espaciales.

Los problemas que al respecto afloran a la hora de llevar a cabo una interpolación espacial convencional son varios:

- Datos escasos y con representatividad espacial limitada. Ello puede deberse a varias causas tales como una distribución espacialmente sesgada ${ }^{1}$, una cifra de puntos muestrales insuficiente, una escala de muestreo inapropiada, i.e. la variabilidad espacial del fenómeno se produce a una escala espacial bastante mayor (esto es, a distancias pequeñas) que la del muestreo (esto es, con distancias grandes entre puntos muestrales), etc. Todo ello puede ocasionar, y de hecho así se ha comprobado en algunos casos, una base deficiente para acometer con éxito y credibilidad la labor de estimación.

- Las técnicas de interpolación espacial pueden considerarse a la vez herramientas simples y sofisticadas. Resultan algo simples, conceptualmente hablando, si se considera el número de variables o factores que se integran en la fórmula de interpolación (a menudo, se circunscriben al valor, la distancia, la ponderación y la orientación-posición relativa entre los puntos muestrales), excluyendo otros condicionantes básicos del fenómeno a estimar. Por ejemplo, en el caso de la polución del aire podrían citarse los tipos de tiempo y la estabilidad/ inestabilidad atmosférica asociada, los vientos, los mecanismos de dispersión espacial de las emisiones, etc. Ello las hace algo limi-

1 La ubicación de las estaciones de medida es un asunto científicamente crítico y políticamente muy sensible, como recuerda Monmonier (1997, cap. 8, especialmente p. 151 y ss.). Aunque existen regulaciones al respecto en diversos países, e. g. los EEUU o la UE, su grado de representatividad espacial, a efectos de usar tal muestra espacial para estimaciones, siempre resulta muy discutible. 
tadas en sus capacidades. A la vez resultan sofisticadas, en parte por su formulación matemática, de complejidad desigual, y en parte, y sobre todo, por requerir decisiones meditadas (no automáticas) para especificar bastantes parámetros de las fórmulas, cuya influencia sobre los resultados aparecen como decisivas. Por ello, deviene imprescindible un manejo inteligente de dichas técnicas, ya que han de subsumir muchos factores implícitos afectando al fenómeno espacial a estimar.

- Las estimaciones espaciales son de difícil validación, salvo en los escasos puntos muestrales.

- Los procesos generadores de patrones espaciales son complejos y, además, los mecanismos operando en cada tipo de proceso presentan bastantes especificidades. Por ello, afinar en la capacidad estimadora requiere un buen conocimiento empírico del fenómeno y del entorno geográfico en cuestión, para evaluar también los resultados con criterios contextuales o cualitativos.

Por todo lo expuesto parece aconsejable, como objetivo general, avanzar hacia el establecimiento de protocolos procedimentales que mejoren las estimaciones, dadas las circunstancias tan adversas de partida.

En este trabajo se describe una estrategia diseñada para estimar la concentración de dióxido de nitrógeno $\left(\mathrm{NO}_{2}\right)$ en la atmósfera de dos ciudades españolas, con unas condiciones ambientales muy diferentes; Barcelona con graves problemas de contaminación y Santa Cruz de Tenerife con buena calidad ambiental. $\mathrm{El} \mathrm{NO}_{2}$ es uno de los contaminantes que la directiva Europea (2008/50/CE) fija como objeto de evaluación por sus impactos sobre la atmósfera. Según el Informe de la Agencia Europea de Medio Ambiente sobre calidad del aire en Europa 2012, el porcentaje de población urbana de la UE expuesta a $\mathrm{NO}_{2}$ excesivo oscila entre el 6 y el 12\% (EEA, 2012). La mayor parte del $\mathrm{NO}_{2}$ proviene de los procesos de combustión de los motores de los vehículos, y en su mayoría interviene en diversos procesos químicos atmosféricos, dando lugar a la formación de ozono troposférico cuando reaccionan con compuestos orgánicos volátiles en presencia de radiación solar, o es la fuente principal de los aerosoles de nitrato, que constituyen una parte importante de las $\mathrm{PM}_{2,5}$, las más dañinas para la salud. $\mathrm{Al}$ inhalarse afecta al tracto respiratorio, inhibiendo algunas funciones pulmonares y mermando la resistencia a infecciones. Las altas concentraciones afectan especialmente a niños y asmáticos, mientras que la exposición continuada a bajas concentraciones se asocia al incremento de enfermedades respiratorias crónicas y a la disminución de la capacidad pulmonar.

En el apartado siguiente se resumen los antecedentes próximos del problema abordado, para luego presentar los datos y métodos utilizados. En la sección de resultados se describen los hallazgos más relevantes en cada una de las fases de la metodología y se concluye con un balance y valoración de la contribución realizada.

\section{ANTECEDENTES Y ESTADO DE LA CUESTIÓN}

El presente trabajo se inscribe dentro de una línea de investigación sobre la calidad del aire en las ciudades y las injusticias socio-ambientales derivadas, en cuyo marco se abordan cuestiones de tipo metodológico, como es comparar los resultados de los métodos de interpolación, analizar las diferencias y similitudes que emergen en los patrones espaciales 
obtenidos con las distintas técnicas, valorar su grado de confianza y dirimir cuáles son los más adecuados, teniendo en cuenta criterios estadísticos y geográficos.

Para el caso específico de las inequidades ambientales en la ciudad de Madrid, por ejemplo, Moreno y Cañada (2007) y Cañada et al. (2011) generaron estimaciones espaciales de contaminantes como el dióxido de azufre y las partículas en suspensión respectivamente y la exposición potencial a ellos de distintos grupos de renta. Con ese mismo fin Moreno (2013) ha evaluado la exposición de la población de Madrid al dióxido de nitrógeno, usando superficies estimadas de dicho contaminante dentro de la ciudad. Obviamente, la credibilidad y el rigor de tales estudios depende de la capacidad de las técnicas de predicción para generar resultados continuos espacialmente y confiables. Cañada (2012) describe y valora la situación y los cambios producidos en los niveles de $\mathrm{NO}_{2}$ en Madrid, en el periodo comprendido entre 2001-2011, contemplando si el cambio en la ubicación y en la reducción del número de estaciones que se produce durante ese periodo afecta decisivamente a los resultados de los valores estimados de $\mathrm{NO}_{2}$ mediante interpolación.

Son muchos los autores que comparan los diferentes métodos de interpolación / estimación espacial (Hengl, 2009; Babak y Deutsch, 2009; Mueller et al., 2004; Wong et al., 2004; Diem y Comrie, 2002). Li y Heap ( 2008, 2011) realizan una revisión de estos métodos y señalan los factores que más les afectan: la densidad del muestreo, la distribución espacial de la muestra, el agrupamiento, el tipo de superficie, la varianza de los datos, la normalidad, la resolución espacial buscada, etc. De Mesnard (2013) reseña abundantes estudios metodológicos sobre el problema de la interpolación espacial con la técnica IDW, recogiendo la diversidad de radios de distancia que se han adoptado para generar estimaciones en puntos nuevos a partir de los datos de las estaciones de medición, desde unas decenas de metros hasta más de $100 \mathrm{~km}$ (id. p. 460-461), así como el variable número de estaciones incluidas en la fórmula de cálculo (desde tres o cuatro hasta diez). Adicionalmente, y más en concreto, ha examinado las cuestiones relacionadas con el exponente afectando a la distancia (y por ende a los pesos usados en la fórmula) para estimar niveles de contaminación de diversos tipos tales como las radiaciones electromagnéticas, polución en ríos, penachos («plumes») derivados de focos puntuales, nubes (ráfagas) explosivas, etc. Para el caso de la contaminación aérea el autor recomienda que tal exponente sea 2 ó 3. Babak y Deutsch (2009: 544) realizan una nueva aportación al método IDW para encontrar el óptimo valor de p y el óptimo número de vecinos usados en la estimación. Consiste en tener en cuenta la estacionariedad de la media y de la varianza y en aplicar un modelo de variograma. Luego realizan un estudio comparativo del Kriging frente al IDW y concluyen con la preferencia del segundo.

Una diferencia crucial en los métodos de interpolación es el modo de determinar los pesos para los puntos muestrales. Si se utilizan métodos deterministas, como la media ponderada por el inverso de la distancia (IDW), los pesos dependen de la distancia entre las estaciones, elevándose esa distancia a un exponente arbitrario e independiente con respecto a los datos, aunque en la realidad no hay tal indefinición, pues su valor depende de factores como la orografía, los vientos dominantes, etc. que condicionan la concentración del contaminante. Por el contrario, el Kriging, denominado interpolador óptimo, utiliza el semivariograma para determinar los pesos, que dependen de la autocorrelación espacial de los datos medidos en las estaciones utilizadas, por lo tanto los pesos son dependientes del valor de los propios datos (De Mesnard, 2013). 
En resumen, no se cuenta con demasiadas orientaciones o reglas para operacionalizar los modelos en casos concretos (lugares y tipo de fenómeno), pues a la multiplicidad y variedad de condicionantes de éstos, se añaden, como hemos señalado antes, las limitaciones de la información disponible y, en particular, la representatividad del muestreo espacial.

Con ello en mente, y partiendo de las peculiaridades y posibilidades de los modelos de interpolación, los escenarios locales elegidos, y los datos disponibles sobre contaminación y sus factores condicionantes en unos contextos geográficos conocidos, se ha decidido ensayar una secuencia de etapas para mejorar ciertas decisiones técnicas a la hora de interpolar. Ellas conciernen a la determinación de la posible tendencia o anisotropía espacial en los datos observados, a la elección del modelo de interpolación y al establecimiento de parámetros del mismo (i.e. número de estaciones a incluir en la fórmula de cálculo, tamaño, forma, orientación y sectorización del área de búsqueda de estaciones vecinas a incluir, exponente de los pesos a aplicar a las estaciones próximas, etc.).

El plan de trabajo ha consistido en la ejecución de una serie de pruebas, según una estrategia de ensayo-error selectiva, que intentará lograr, tras un limitado número de operaciones, un resultado técnica y ambientalmente aceptable con los datos disponibles. En el apartado siguiente se describe y justifica la metodología y datos utilizados, para luego presentar, comparar y valorar los resultados experimentales. Tras ello se enuncian sintéticamente las conclusiones alcanzadas.

\section{DATOS Y MÉTODOS}

\subsection{Las fuentes de información}

Los datos ambientales para Barcelona proceden de la Xarxa de Vigilància i Previsió de la Contaminació Atmosfèrica ((XVPCA), dependiente de la Dirección General de Qualitat Ambiental, Departament de Territori i Sostenibilitat de la Generalitat de Catalunya. De acuerdo con el RD 102/2011, el estudio de la calidad del aire se realiza por zonas definidas con unas características similares; las estaciones utilizadas en este trabajo pertenecen al municipio de Barcelona, pero dado el número reducido de las mismas (6) también se han utilizado otras 6 provenientes de municipios limítrofes; todas están dentro de la zona 1 de calidad de aire, denominada Área de Barcelona, con una población de 2.858.770 habitantes según el Anuario Estadístico de Cataluña de 2010. Los datos de Santa Cruz de Tenerife proceden de la Consejería de Educación, Universidades y Sostenibilidad del Gobierno de Canarias. Se han utilizado nueve estaciones ubicadas en el municipio de Santa Cruz de Tenerife y tres estaciones de un municipio próximo.

La variable ambiental seleccionada ha sido la concentración media anual de $\mathrm{NO}_{2}$ del año 2010 para Barcelona y 2012 para Santa Cruz de Tenerife. Se recuerda que el nivel medio anual admisible por la legislación española, comunitaria y por la Organización Mundial de la Salud se sitúa en $40 \mu \mathrm{g} / \mathrm{m}^{3}$.

Como cartografía digital básica se han usado las delimitaciones espaciales de los distritos y de los términos municipales del INE. Si bien el análisis se ha circunscrito a la zona urbana poblada, excluyendo los territorios claramente no residenciales y los periféricos no urbanizados (véase figura 1), tras una labor de identificación de usos del suelo apoyada en el Corine Land Cover 2006 y en imágenes aéreas recientes. 


\subsection{Metodología para la interpolación espacial de la contaminación del aire}

De cara a los objetivos planteados se ha diseñado un procedimiento con varias etapas. En la primera se realiza un análisis exploratorio de los datos muestrales de contaminación, donde se obtienen las propiedades de centralidad, dispersión y forma de su distribución.

Figura 1

LOCALIZACIÓN DE LAS ESTACIONES DE CONTAMINACIÓN Y DEL ÁREA URBANA POBLADA DE BARCELONA Y DE SANTA CRUZ DE TENERIFE

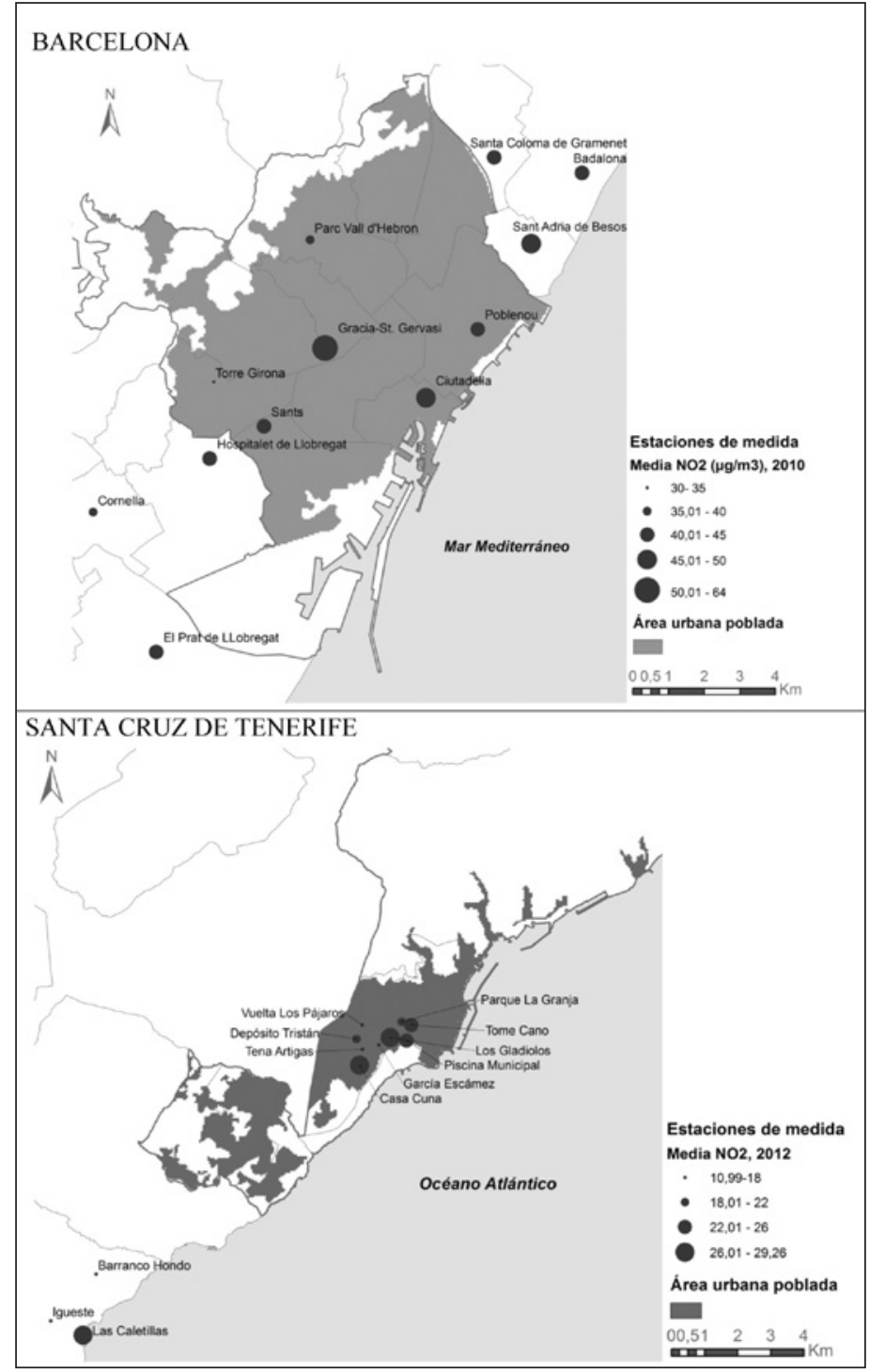


En la segunda etapa se aplica un análisis estructural de los mismos, mediante la construcción del semivariograma, para desvelar si la semivarianza cambia sólo con la distancia o también en función de la dirección. El objetivo ahora estriba en obtener una serie de estadísticos como el alcance, la meseta o el nugget e identificar las direcciones de máxima y mínima continuidad espacial; se trata de encontrar la forma de vecindad, bien un círculo o una elipse, para luego elegir los puntos que se utilizarán para estimar el valor de cada lugar. En caso de detectar anisotropía espacial se definiría una elipse cuyos ejes mayor y menor reflejasen adecuadamente la misma. En caso de no detectar anisotropía se optaría por el modelo isotrópico, en el que sólo se tendría en cuenta la variación de los valores en función de la distancia.

Otro instrumento de exploración utilizado en esta segunda etapa es el Global Polynomial Interpolation (GPI), también llamado análisis de superficie de tendencia, un modelo de interpolación determinista e inexacto que orienta sobre el patrón espacial general de la variable y que ayuda también en la tercera fase del análisis, de interpolación propiamente dicha, a escoger los vecinos (puntos muestrales) con un círculo o una elipse y a dar dimensiones a los ejes de la misma. Al respecto se han usado fundamentalmente polinomios de grados 1, 2 y 3 por no ser necesarios más. Existe abundante bibliografía sobre este método (e.g. Goodin et al., 1979).

En la tercera etapa del análisis se aplican ya los dos métodos de interpolación seleccionados, uno, determinista, exacto y local, la media ponderada por el inverso de la distancia (IDW), y otro, un método geoestadístico y analítico, el Kriging ordinario, que tiene en cuenta la autocorrelación espacial de la variable a interpolar. El objetivo ahora es confrontar sus resultados y dirimir la aceptabilidad de cada uno de ellos, adoptando criterios estadísticos

convencionales, como la bondad de ajuste, y otros geográf
Ambos métodos utilizan la misma fórmula de cálculo: $Z\left(s_{0}\right)=\sum_{i=1}^{N} \lambda_{i} * Z\left(s_{i}\right)$, donde $\mathrm{Z}\left(\mathrm{s}_{0}\right)$ es el valor que se intenta predecir para el lugar $\mathrm{s}_{0}$, $\mathrm{N}$ es el número de puntos muestrales alrededor del lugar que se va a predecir y que serán tenidos en cuenta en el cálculo, $\lambda_{i}$ es el peso asignado a cada punto muestral y $\mathrm{Z}\left(\mathrm{s}_{\mathrm{i}}\right)$ es el valor observado en el lugar $\mathrm{s}_{\mathrm{i}}$.

Lo que diferencia a ambos métodos es el procedimiento de cálculo de los pesos asignados a los valores muestrales. En el caso del IDW los pesos se determinan mediante la fórmula: $\lambda_{i}=d_{i 0}^{-p} / \sum_{i=1}^{N} d_{i 0}^{-p}$, donde $\mathrm{d}_{\mathrm{i} 0}$ es la distancia entre el lugar de predicción $\mathrm{s}_{0} \mathrm{y}$ cada lugar muestral $\mathrm{s}_{\mathrm{i}}$, a medida que la distancia se hace más grande, el peso es reducido por un factor $\mathrm{p}$ (Cañada, 2007: 793; Wong et al., 2004: 406).

En el Kriging ordinario los pesos se calculan mediante la función del semivariograma, cuyo ajuste más frecuente se realiza mediante el modelo esférico, que adopta la siguiente expresión:

$$
\begin{aligned}
& \gamma(h)=\left\{\theta_{s}\left[\frac{3}{2} \frac{h}{\theta_{r}}-\frac{1}{2}\left(\frac{h}{\theta_{r}}\right)^{3}\right] \quad \text { si } 0 \leq h \leq \theta_{r}\right. \\
& \gamma(h)=\theta_{s} \quad \operatorname{si\theta }_{r}<h
\end{aligned}
$$

donde $\theta_{\mathrm{s}} \geq 0$ es el partial sill (meseta parcial) y $\theta_{\mathrm{r}} \geq 0$ es el range (alcance) (Cañada, 2007: 840; Wong et al., 2004: 407; Krivoruchko, 2011). 
La aplicación de estas técnicas se ha realizado mediante la extensión Geostatistical Analyst de ArcGIS 10.1. En ambos casos, el análisis se ha iniciado con los parámetros por defecto que presentaba el programa, para variarlos en repetidas iteraciones hasta alcanzar los menores errores en la predicción: mean error (ME), media de los errores próxima a 0 y root mean square error (RMSE), error cuadrático medio más bajo.

El objetivo final consistía en dilucidar qué método de interpolación proporcionaba los menores errores y a la vez presentaba una distribución espacial del contaminante más compatible y coherente con el marco territorial y la estructura urbana local.

Con ambas técnicas la búsqueda de vecindad comenzó con un círculo (modelo isotrópico), para después pasar a una elipse (modelo anisotrópico), porque reflejaba mejor el reparto espacial del contaminante al ajustarse más a la orografía de los municipios y a los vientos dominantes. Se ha explorado sistemáticamente la dirección de máxima y mínima continuidad espacial de la variable, la orientación del eje principal de anisotropía, el número de cuadrantes en que se divide la elipse, la rotación u orientación de dichos cuadrantes, el número de vecinos a incluir y el valor de p en el caso específico del IDW.

El resultado de la interpolación es una superficie geostadística que hay que convertir a capa raster, habiéndose seleccionado una resolución de 50 metros. Con posterioridad, y mediante álgebra de mapas, la capa raster se ha recortado con la capa que representa la zona urbana poblada. Los estadísticos descriptivos de esta última capa recortada son los que se utilizarán en el próximo apartado para comprobar y valorar en qué medida los valores pronosticados de $\mathrm{NO}_{2}$ se aproximan a los observados.

\section{ANÁLISIS DE LOS RESULTADOS}

\subsection{Caso de Barcelona}

\subsubsection{Exploración estadística de los datos muestrales de $\mathrm{NO}_{2}$}

La descripción univariada de las medias anuales de dióxido de nitrógeno en Barcelona (tabla 1) muestra una gran concentración de valores observados en torno a la media, de ahí el alto valor de la kurtosis. Su asimetría es positiva, aunque con dos colas definidas, que representan el valor más bajo y el más alto de la serie (figura 2A). La distribución de los datos tampoco es normal (figura 2B) y muestra una tendencia de aumento de los valores hacia la parte central del municipio y una disminución desde el centro hacia el norte y sur y hacia el este y el oeste (véase figura $2 \mathrm{C}$ ).

La superficie del semivarigorama (figura 2D), obtenida a partir del semivariograma empírico, confirma que la semivarianza de los datos de dióxido de nitrógeno en Barcelona presenta diferencias espaciales, tanto en función de la distancia como en función de la dirección. Se puede observar que es más alta en la zona central y que disminuye hacia los extremos noreste y suroeste.

\subsubsection{Análisis estructural}

Para desvelar la estructura espacial de los datos se ha utilizado el GPI y el ajuste teórico del semivarigrama empírico. La superficie calculada con el GPI de tercer grado adopta la 
Tabla 1

ESTADÍSTICOS DE LOS DATOS MUESTRALES DE NO ${ }_{2}$ EN BARCELONA $2010 \mu \mathrm{g} / \mathrm{m}^{3}$

\begin{tabular}{|l|c|}
\hline & BARCELONA \\
\hline Media & 42,9 \\
\hline Mínimo & 30 \\
\hline Máximo & 64 \\
\hline Desv. estándar & 8 \\
\hline Asimetría & 1,29 \\
\hline Kurtosis & 5,4 \\
\hline Q1 & 40 \\
\hline Q2 & 41 \\
\hline Q3 & 45,5 \\
\hline N & 12 \\
\hline
\end{tabular}

Fuente: Elaboración propia.

forma de elipse, con el eje más grande en sentido NE-SW y el eje más corto en dirección NW-SE (figura 3A). Los valores estimados son más elevados en la zona central de la elipse y disminuyen de manera gradual en sentido NE-SW; sin embargo la disminución es más rápida desde el centro en sentido NW-SE. La utilización de GPI permite constatar espacialmente el comportamiento anisotrópico del dióxido de nitrógeno en Barcelona, a partir de los datos registrados en las estaciones.

Figura 2

GRÁFICOS DEL ANÁLISIS EXPLORATORIO DE DATOS, BARCELONA 2010

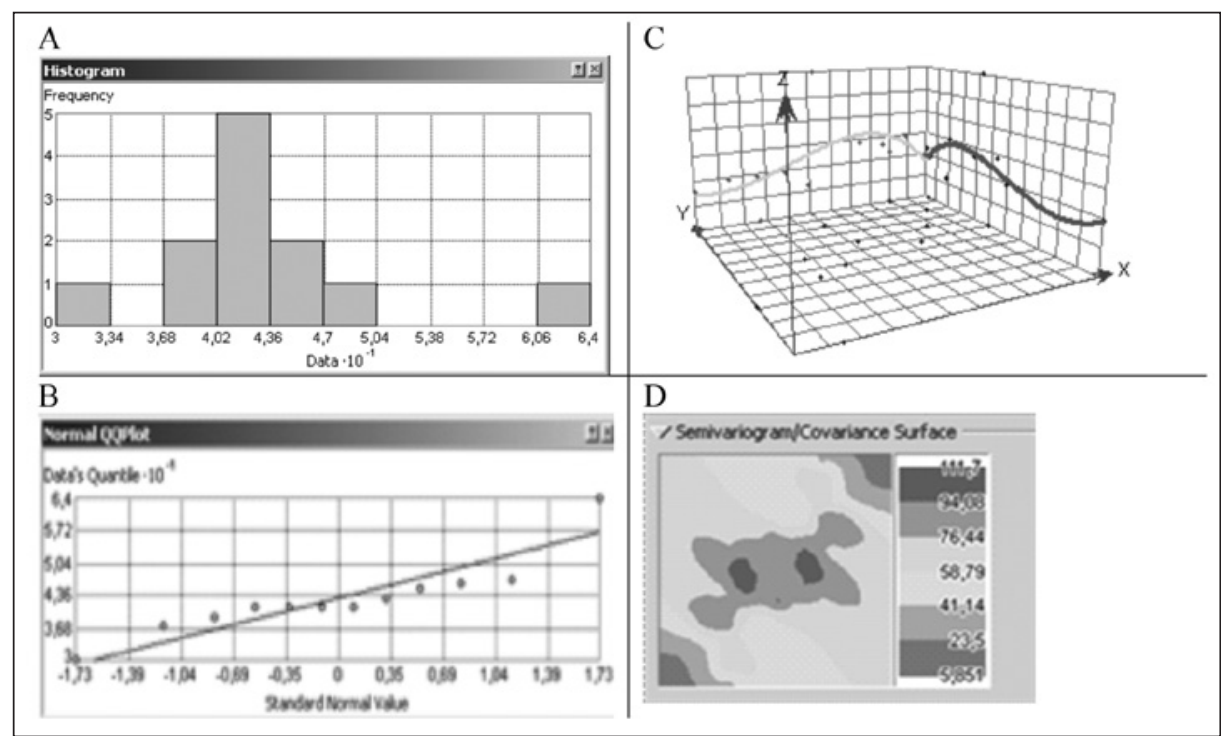




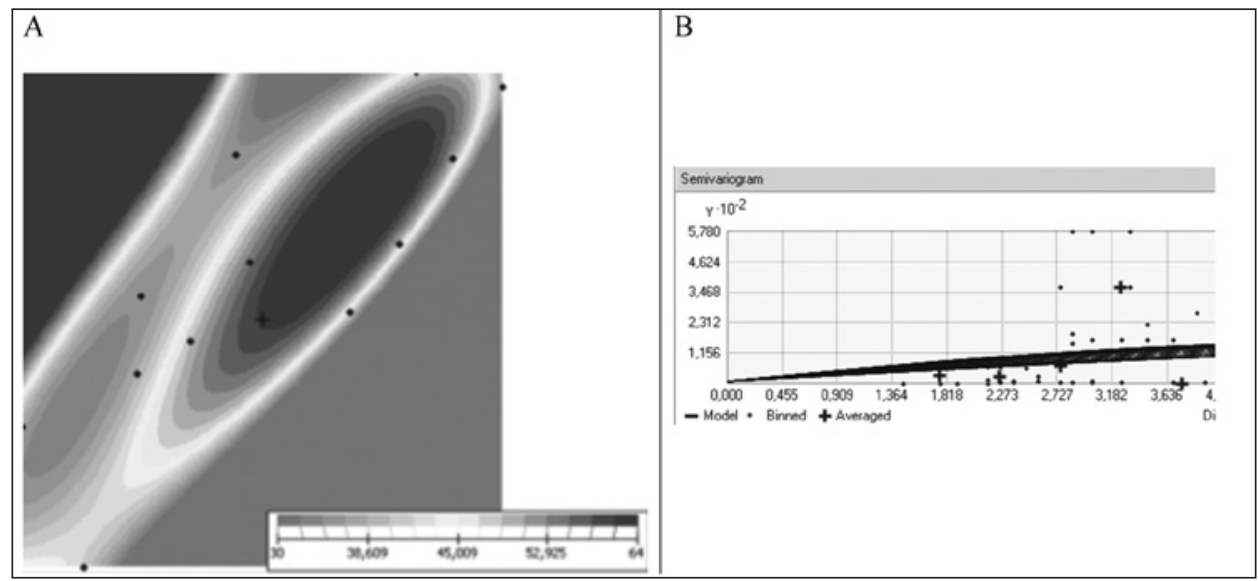

Para determinar de manera objetiva la orientación de la elipse y el factor de anisotropía se ha ajustado una función al semivariograma empírico (figura 3B), cuyo modelo ha adoptado finalmente los siguientes valores: 7,8489* Nugget $+147,64 *$ Spherical $(9000,5000,35,0)$, agrupando los datos en 10 intervalos con una amplitud de $500 \mathrm{~m}$. Es decir, la distancia a la que la varianza de la variable se estabiliza es $9000 \mathrm{~m}$ en sentido NE-SW (35 $)$; por el contrario esa varianza se estabiliza a $5000 \mathrm{~m}$ en sentido NW-SE. Se recuerda que con estos parámetros se ha obtenido un menor RMSE y un valor RMSSE más próximo a 1. Estos valores se han utilizado como punto de partida en la interpolación con IDW, cuando se ha aplicado el modelo anisotrópico como forma de búsqueda de vecindad, puesto que se ha comprobado que los valores de la semivarianza eran más continuos a lo largo de la dirección NE-SW que en la dirección NW-SE.

Las iteraciones realizadas con la técnica del IDW usando diferentes parámetros han sido numerosas hasta conseguir unos resultados satisfactorios. En concreto se han manejado unas dimensiones entre $11000 \mathrm{~m}$ y $9000 \mathrm{~m}$ para el eje mayor y entre $5000 \mathrm{~m}$ y $4000 \mathrm{~m}$ para el eje menor. También se han utilizado variaciones en el número de vecinos, que han oscilado entre 7 y 4 y entre 6 y 4 y el valor de p ha fluctuado entre 1 y 3,7. En cuanto a la orientación de la elipse se ha probado con un amplio abanico de valores que oscilan entre $28^{\circ}$ y $60^{\circ}$. La solución que proporcionaba los menores errores en la estimación de $\mathrm{NO}_{2}$ no era aceptable ya que, al comprobar la plasmación espacial de los valores estimados, se constataron deficiencias serias, tales como saltos bruscos (escalones) en la parte noroeste del mapa, cuya paliación se logró en ensayos adicionales, recurriendo a valores más altos de p (la solución aceptada es la representada en la figura 5B).

\subsubsection{Interpolación espacial de la polución por $\mathrm{NO}_{2}$ y comparación de soluciones}

La aplicación de las técnicas previas de exploración ha corroborado el comportamiento anisotrópico del $\mathrm{NO}_{2}$ en Barcelona, es decir, que en una determinada dirección, generalmente 
la NNE-SSW, hay una mayor continuidad espacial de la variable, mientras que en la dirección perpendicular, WNW-ESE, la continuidad espacial es menor, lo que significa que los cambios de la semivarianza son mucho más bruscos en menores distancias. Las razones de este comportamiento en el caso de Barcelona parecen obedecer a la topografía de la ciudad, asentada en una plataforma de ligera pendiente, encajonada entre el mar por el este y la Sierra de Collserola al noroeste, y predominando los vientos de componente norte que dispersan más el contaminante en el sentido del eje de máxima continuidad espacial.

\section{a) Aplicación del Kriging y del IDW}

El método del Kriging se aplicó inicialmente con los parámetros por defecto: un círculo como búsqueda de vecindad, de un diámetro de $4878 \mathrm{~m}$, un valor de p igual a 2 y un número de vecinos entre 15 como máximo y 10 como mínimo. En este primer intento la media de los errores fue de 0,36 y el RMSE 8,78. El patrón espacial, sin embargo, resultaba poco plausible para ese contexto geográfico (algunas estimaciones estaban alejadas de las expectativas razonables). Tras dos fases sucesivas, donde se cambiaron el diámetro del círculo, que aumentó a 5000 m, el número de intervalos, que se redujo a 10, y la amplitud de los mismos, que bajó hasta $500 \mathrm{~m}$, se consiguió un patrón espacial más acorde con la trama urbana, aunque no en su totalidad, si bien aumentó un poco el RMSE (véase tabla 2).

Tabla 2

PARÁMETROS UTILIZADOS Y BONDAD DE AJUSTE CON EL MÉTODO DE INTERPOLACIÓN KRIGING (BARCELONANO 2 2010)

\begin{tabular}{|l|c|c|c|c|c|c|c|}
\hline TÉCNICA & \multicolumn{3}{|c|}{ PARAMETROS DEL MODELO } & \multicolumn{2}{|c|}{ VALIDACIÓN CRUZADA } \\
\hline & $\begin{array}{c}\mathrm{N}^{\circ} \\
\text { vecinos }\end{array}$ & $\begin{array}{c}\text { Forma/ } \\
\text { sectores/ } \\
\text { rotación/ } \\
\text { tamaño/m }\end{array}$ & Ángulo & $\begin{array}{c}\text { Factor de } \\
\text { anisotropía }\end{array}$ & $\begin{array}{c}\bar{X} \\
\text { errores }\end{array}$ & RMSE & RMSSE \\
\hline $\begin{array}{l}\text { KRIGING } \\
\text { ISOTRÓPICO }\end{array}$ & $5 / 2$ & $\begin{array}{l}\text { Círculo, } 4 \\
\text { partes, } 45^{\circ}, \\
5000 \mathrm{~m}\end{array}$ & 0 & 1 & 0,21 & 9,19 & 0,87 \\
\hline $\begin{array}{l}\text { KRIGING } \\
\text { ANISOTRÓPICO }\end{array}$ & $5 / 2$ & $\begin{array}{l}\text { Elipse, } 4 \\
\text { partes, } 45^{\circ}, \\
9000 / 5000\end{array}$ & 35 & 1,8 & 0,47 & 8,94 & 0,89 \\
\hline
\end{tabular}

Fuente: Elaboración propia.

El modelo de Kriging con anisotropía, tras dos iteraciones, alcanzó mejores resultados, no sólo en la validación del modelo, con un menor error cuadrático medio (RMSE) de 8,94 y un error cuadrático medio estandarizado más próximo (RMSSE) a la unidad $(0,89)$, sino también en la configuración espacial del contaminante. Sin embargo como se ha comentado con anterioridad, al producir una mayor suavizado, ha aumentado la distancia entre los valores medios estimados y observados (véase tabla 4). 
En la tabla 3 figuran los parámetros del modelo isotrópico y anisotrópico con la técnica IDW para el municipio de Barcelona. También se incluyen los estadísticos que permiten validar el modelo, la media de los errores y el error cuadrático medio (RMSE). Al comparar los errores de la predicción (RMSE) se observa que son un poco mayores en el modelo anisotrópico seleccionado que en el isotrópico, lo que contradice el criterio estadístico usual, sin embargo, como se verá más adelante, teniendo en cuenta la plasmación espacial del contaminante, resulta más ajustado a la realidad geográfica local la solución del modelo anisotrópico. En la misma tabla viene reflejado el modelo anisotrópico con el mejor ajuste, es decir, un menor RMSE, pero que fue rechazado por ser incoherente con la estructura urbana y presentar cambios bruscos de tendencia. Por otro lado, unos errores cuadráticos medios más reducidos con el IDW que con el Kriging avalan mejor a aquél como método para interpolar.

Tabla 3

PARÁMETROS UTILIZADOS Y BONDAD DE AJUSTE CON EL MÉTODO DE INTERPOLACIÓN IDW (BARCELONA NO 2 2010)

\begin{tabular}{|c|c|c|c|c|c|c|c|}
\hline \multirow[t]{2}{*}{ TÉCNICA } & & \multicolumn{4}{|c|}{ PARAMETROS DEL MODELO } & \multicolumn{2}{|c|}{$\begin{array}{c}\text { VALIDACIÓN } \\
\text { CRUZADA }\end{array}$} \\
\hline & $\mathrm{p}$ & $\begin{array}{c}\mathrm{N}^{\circ} \\
\text { vecinos }\end{array}$ & $\begin{array}{l}\text { Forma / } \\
\text { sectores / } \\
\text { rotación / } \\
\text { tamaño/ m }\end{array}$ & Ángulo & $\begin{array}{c}\text { Factor de } \\
\text { anisotropía }\end{array}$ & $\underset{\text { errores }}{\bar{X}}$ & RMSE \\
\hline $\begin{array}{l}\text { IDW } \\
\text { ISOTRÓPICO }\end{array}$ & 1 & $12 / 6$ & $\begin{array}{c}\text { Círculo, } 4 \\
\text { partes, } 45^{\circ} \text {, } \\
4878 \\
\end{array}$ & 0 & 1 & 0,30 & 8,53 \\
\hline $\begin{array}{l}\text { IDW } \\
\text { ANISOTRÓPICO } \\
\text { CON MENOR } \\
\text { RMSE }\end{array}$ & 2,25 & $7 / 4$ & $\begin{array}{c}\text { Elipse, } 4 \\
\text { partes, } 45^{\circ}, \\
9000 \\
/ 4000\end{array}$ & 31 & 2,25 & 0,48 & 8,48 \\
\hline $\begin{array}{l}\text { IDW } \\
\text { ANISOTRÓPICO }\end{array}$ & 3,6 & $7 / 4$ & $\begin{array}{c}\text { Elipse, } 4 \\
\text { partes, } 45^{\circ}, \\
9000 / \\
5000\end{array}$ & 35 & 1,8 & 0,35 & 8,77 \\
\hline
\end{tabular}

Fuente: Elaboración propia.

En síntesis, al comparar los estadísticos descriptivos de los valores observados de contaminación de $\mathrm{NO}_{2}$ con los estimados (zona urbana poblada exclusivamente) por las dos técnicas se constata lo siguiente (tabla 4):

- La media de los datos estimados con la técnica IDW está más próxima a los datos empíricos e incluso coincide con ella en el caso del modelo isotrópico.

- Los valores máximos y mínimos pronosticados con el IDW modelo anisotrópico coinciden con los observados.

- Con el Kriging la media de los datos estimados es superior a la de los observados. En el Kriging modelo anisotrópico es de $45,3 \mu \mathrm{g} / \mathrm{m}^{3}$ y en el isotrópico de $44,8 \mu \mathrm{g} / \mathrm{m}^{3}$, 
frente a $42,9 \mu \mathrm{g} / \mathrm{m}^{3}$ de media en los datos observados. Sin embargo el valor máximo lo ha reducido un poco, $62 \mu \mathrm{g} / \mathrm{m}^{3}$ frente a $64 \mu \mathrm{g} / \mathrm{m}^{3}$ y un mínimo nueve décimas más alto. Así pues recorta los valores altos y eleva los valores bajos, ocasionando un mayor suavizado que resulta inconveniente.

- Considerando, pues, los estadísticos expuestos es preferible el modelo IDW frente al Kriging.

- Pero además de estos criterios numéricos, hay que tener en cuenta el patrón espacial resultante del modelado. Siempre el modelo anisotrópico genera una configuración más plausible, pasando de las zonas donde hay una mayor concentración del contaminante a las zonas de menor concentración de modo gradual, sin saltos abruptos (escalones). Ello respalda mejor al modelo anisotrópico frente al isotrópico.

b) Comparación de los patrones de $\mathrm{NO}_{2}$ interpolados

Como resultado del modelado del $\mathrm{NO}_{2}$ se han obtenido cuatro mapas de isopletas, dos para cada método de interpolación, en los que se muestran cinco intervalos realizados de modo manual para poder diferenciar qué partes del municipio incumplían el valor límite medio anual establecido por la legislación $\left(40 \mu \mathrm{g} / \mathrm{m}^{3}\right)$.

Tabla 4

COMPARACIÓN DE LOS ESTADÍSTICOS DESCRIPTIVOS DE AMBOS MÉTODOS Y MODELOS

\begin{tabular}{|l|c|c|c|c|c|}
\hline \multirow{2}{*}{$\begin{array}{l}\text { ESTADÍSTICOS } \\
\text { DESCRIPTIVOS }\end{array}$} & \multirow{2}{*}{$\begin{array}{c}\text { DATOS } \\
\text { OBSERVADOS }\end{array}$} & \begin{tabular}{c} 
DATW \\
\cline { 3 - 6 }
\end{tabular} & $\begin{array}{c}\text { ISOTRÓPICO } \\
\text { ANISOTRÓPICO }\end{array}$ & $\begin{array}{c}\text { KRIGING } \\
\text { ISOTRÓPICO }\end{array}$ & $\begin{array}{c}\text { KRIGING } \\
\text { ANISOTRÓPICO }\end{array}$ \\
\hline Media & 42,9 & 42,9 & 44,4 & 44,8 & 45,3 \\
\hline Desviación típica & 8 & 1,7 & 7,5 & 5,8 & 6,7 \\
\hline Máximo & 64 & 63,6 & 64 & 63,9 & 62 \\
\hline Mínimo & 30 & 30,62 & 30 & 30,05 & 30,9 \\
\hline Amplitud & 34 & 33 & 34 & 33,85 & 31,1 \\
\hline
\end{tabular}

Fuente: Elaboración propia.

El examen de los dos mapas más representativos, IDW y Kriging (figuras 4B y 5B) con modelos anisotrópicos, muestra una gran extensión que cubre buena parte del centro de la ciudad y zona del puerto, donde se dan las mayores concentraciones de $\mathrm{NO}_{2}$, superiores a 46 $\mu \mathrm{g} / \mathrm{m}^{3}$ y hasta $63 \mu \mathrm{g} / \mathrm{m}^{3}$. Son los barrios de Gracia y parte de St. Gervasi, recorridos por grandes avenidas con tráfico muy intenso, como la Ronda General Mitre, Vía Augusta, Travessera de Dalt, Avenida de la Diagonal. También hay que destacar la contaminación producida por las actividades desarrolladas en las instalaciones del puerto de la ciudad. Desde ese sector la contaminación disminuye de modo gradual, con valores entre 40 y $46 \mu \mathrm{g} / \mathrm{m}^{3}$, tanto hacia el NE, Poblenou, como hacia el NW. El área menos contaminada (valores por debajo de $40 \mu \mathrm{g} /$ $\mathrm{m}^{3}$ ) corresponde a Vall d'Hebron y Torre Girona, zona de transición hacia la Sierra de Collserola, con barrios residenciales y amplios espacios verdes. 
- Patrones espaciales de la polución generados con Kriging isotrópico y anisotrópico

Desde el punto de vista espacial el Kriging arroja patrones algo diferentes con el modelo isotrópico (figura 4A) y el anisotrópico (figura 4B). Se observa un mayor suavizado en el segundo que en el primero, de ahí su menor amplitud, el valor mínimo en ambos es semejante pero el valor máximo es inferior en el modelo anisotrópico (véase tabla 4).

La parte de la ciudad que cumple el límite máximo de concentración media anual, es decir que está por debajo de $40 \mu \mathrm{g} / \mathrm{m}^{3}$, alcanza el 17,27\% (1250,5 ha) en el modelo isotrópico y el 21,26\% (1490 ha) en el modelo anisotrópico; es decir, el 82,73\% y el 78,74\% del territorio respectivamente sobrepasan el umbral de protección de la salud humana (tabla 5).

Tabla 5

SUPERFICIE OCUPADA POR LOS DIFERENTES NIVELES DE NO 2 CON EL MÉTODO KRIGING, BARCELONA 2010

\begin{tabular}{|l|r|r|r|r|}
\hline \multirow{2}{*}{$\begin{array}{c}\text { INTERVALOS } \\
\mu \mathrm{g} / \mathrm{m}^{3}\end{array}$} & \multicolumn{2}{|c|}{$\begin{array}{c}\text { KRIGING } \\
\text { ISOTRÓPICO }\end{array}$} & \multicolumn{2}{c|}{$\begin{array}{c}\text { KRIGING } \\
\text { ANISOTRÓPICO }\end{array}$} \\
\hline & Superficie ha & \multicolumn{1}{c|}{$\%$} & Superficie ha & \multicolumn{1}{c|}{$\%$} \\
\hline$<37$ & 496,25 & 7,08 & 1064,75 & 15,19 \\
\hline $37-40$ & 714,25 & 10,19 & 425,25 & 6,07 \\
\hline $40-43$ & 1395,25 & 19,91 & 713,5 & 10,18 \\
\hline $43-46$ & 1688,75 & 24,10 & 1497,0 & 21,36 \\
\hline$>46$ & 2713,75 & 38,72 & 3307,75 & 47,20 \\
\hline
\end{tabular}

Fuente: Elaboración propia.

Figura 4

PATRONES ESPACIALES DE LA CONTAMINACIÓN POR NO 2 MEDIANTE EL MÉTODO KRIGING ISOTRÓPICO (A) Y ANISOTRÓPICO (B) EN BARCELONA 2010

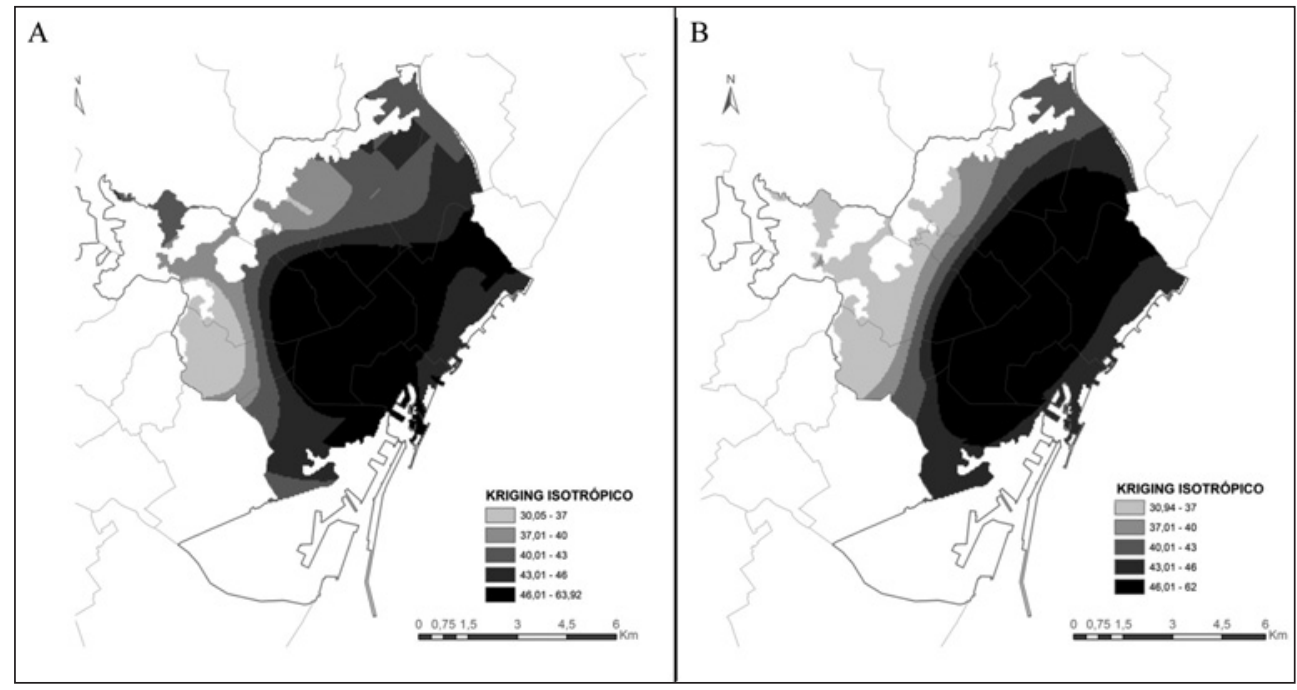


La distribución espacial de la contaminación por $\mathrm{NO}_{2}$ mediante el método IDW utilizando un modelo isotrópico o anisotrópico muestra unos resultados bastantes diferentes entre sí (figura 5). El resultado del modelo isotrópico (figura 5A) muestra los conocidos «ojos de buey», en torno a las estaciones de valores más altos y más bajos, los cuales ocupan una escasa fracción del área urbana. Esto expresa la influencia acusada del valor de un punto de muestreo sobre los de su entorno inmediato. Es un patrón espacial con transiciones muy abruptas, que presenta una gran concentración ( $86 \%$ del municipio, véase tabla 6 ) en torno a los valores centrales 40 y $46 \mu \mathrm{g} / \mathrm{m}^{3}$.

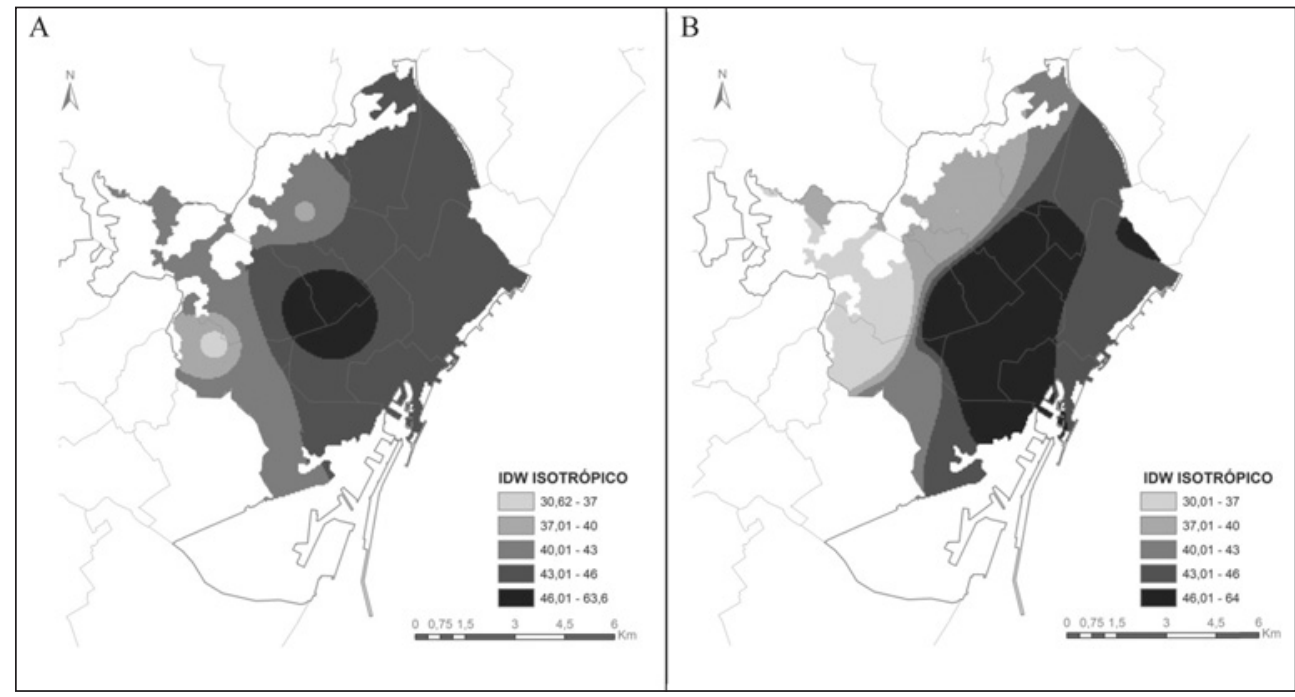

El mapa modelado por el IDW anisotrópico (figura 5 B) muestra una apariencia semejante a la del Kriging anisotrópico, si bien el reparto espacial de los niveles de polución es un poco diferente. El 26,19\% de la zona urbana poblada (aproximadamente 1835,25 ha) cumple el umbral de protección de la salud humana, $40 \mu \mathrm{g} / \mathrm{m}^{3}$, por lo que se considera que tiene una mejor calidad de aire; sin embargo el $73,81 \%$ restante $(5173,15$ ha) lo incumple y presenta peores condiciones ambientales. El área ocupada por los niveles más altos de contaminación, por encima de $46 \mu \mathrm{g} / \mathrm{m}^{3}$ (tabla 6) representa el 33\% (2314,5 ha) y corresponde a las zonas de tráfico más intenso de la ciudad. Desde el centro de la ciudad los niveles de $\mathrm{NO}_{2}$ disminuyen de manera gradual hacia el noroeste, a medida que se asciende hacia la Sierra de Collserola.

La configuración derivada de este modelo no sólo resulta más congruente con el contexto territorial y la estructura urbana, sino que proporciona una mejor bondad de ajuste según los estadísticos descriptivos: una media más reducida que la del Kriging y unos valores máximo y mínimo estimados coincidentes con los observados, por lo que fue el elegido. 
Tabla 6

SUPERFICIE OCUPADA POR LOS DISTINTOS NIVELES DE NO INTERPOLADOS CON EL MÉTODO IDW

\begin{tabular}{|l|r|r|r|r|}
\hline \multirow{2}{*}{$\begin{array}{c}\text { INTERVALOS } \\
\mu \mathrm{g} / \mathrm{m}^{3}\end{array}$} & \multicolumn{2}{|c|}{ IDW ISOTRÓPICO } & \multicolumn{2}{c|}{ IDW ANISOTRÓPICO } \\
\cline { 2 - 5 } & Superficie ha & $\%$ & \multicolumn{1}{c|}{ Superficie ha } & \multicolumn{1}{c|}{} \\
\hline$<37$ & 51,75 & 0,74 & 852,75 & 12,17 \\
\hline $37-40$ & 298,25 & 4,26 & 982,5 & 14,02 \\
\hline $40-43$ & 1769 & 25,24 & 813,5 & 11,61 \\
\hline $43-46$ & 4272,25 & 60,96 & 2045 & 29,18 \\
\hline$>46$ & 617 & 8,80 & 2314,5 & 33,03 \\
\hline
\end{tabular}

Fuente: Elaboración propia.

\subsection{Caso de Santa Cruz de Tenerife}

\subsubsection{Exploración estadística de los datos muestrales de $\mathrm{NO}_{2}$}

Los datos de $\mathrm{NO}_{2}$ no tienen una gran variabilidad, así lo atestigua la pequeña diferencia entre el valor máximo y el mínimo y la baja desviación típica (tabla 7). Su distribución es prácticamente simétrica $(0,01)$, platicurtica (tabla 7 y figura $6 \mathrm{~A}$ ) y casi se ajusta a una distribución normal (figura 6B). Los valores muestran una disminución clara en sentido sureste hacia el noroeste (figura 6C).

La superficie del semivariograma (figura 6D) confirma las diferencias espaciales de la semivarianza en función de la distancia y de la dirección. Los valores más bajos de semivarianza siguen una dirección noroeste-sureste, mientras que desde el centro del municipio dichos valores aumentan hacia el noreste y hacia el suroeste.

Tabla 7

ESTADÍSTICOS DE LOS DATOS MUESTRALES DE NO ${ }_{2}$ EN SANTA CRUZ DE TENERIFE $2012\left(\mu \mathrm{g} / \mathrm{m}^{3}\right)$

\begin{tabular}{|l|c|}
\hline & SANTA CRUZ DE TENERIFE \\
\hline Media & 20,52 \\
\hline Mínimo & 10,99 \\
\hline Máximo & 29,26 \\
\hline Desv. Estándar & 5,51 \\
\hline Asimetría & 0,01 \\
\hline Kurtosis & 1,77 \\
\hline Q1 & 16,04 \\
\hline Q2 & 19,51 \\
\hline Q3 & 25,82 \\
\hline N & 12 \\
\hline
\end{tabular}

Fuente: Elaboración propia. 


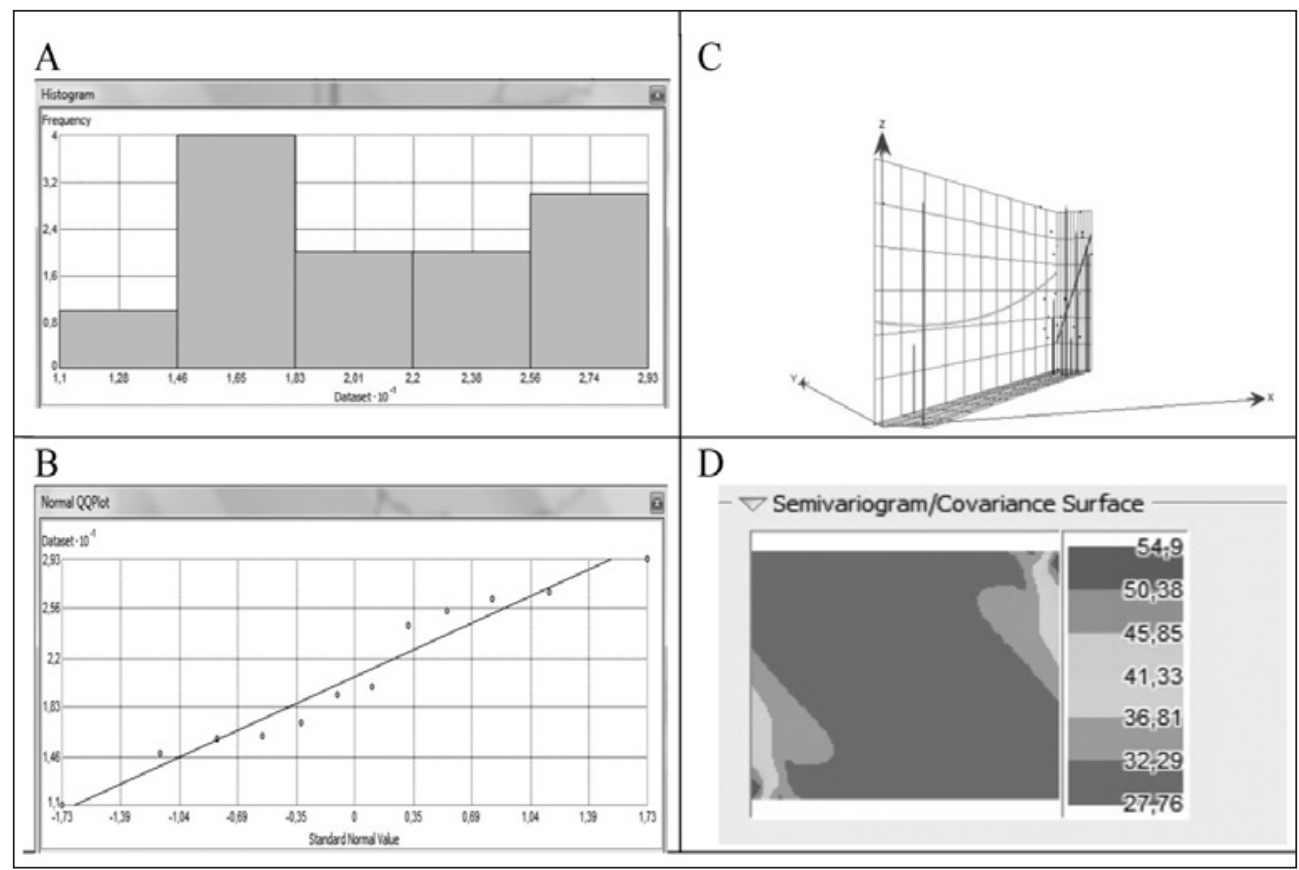

Fuente: Elaboración propia.

\subsubsection{Análisis estructural}

El GPI de primer grado y el ajuste teórico del semivariograma constatan el comportamiento anisotrópico de la media de dióxido de nitrógeno en el municipio de Santa Cruz de Tenerife. El modelo de ajuste del semivariograma empírico (figura 7B) ha adoptado los siguientes valores: $25,239 *$ Nugget $+13,927 *$ Spherical $(15000,3000,43,0)$. Los datos se han agrupado en 12 intervalos con una amplitud de $2000 \mathrm{~m}$. La semivarianza de la variable se estabiliza a 15000 metros en sentido NE-SW $\left(43^{\circ}\right)$ y a $3000 \mathrm{~m}$ en sentido NW-SE.

Lo mismo que en Barcelona el proceso de interpolación con IDW se ha iniciado con los parámetros por defecto que mostraba el programa. Se han realizado numerosas pruebas hasta conseguir resultados satisfactorios tanto en los errores del modelo como en su adaptación espacial a la trama urbana. Las dimensiones de la elipse han oscilado entre los 6000 y 12000 $\mathrm{m}$ para el eje mayor y entre 2000 y $4000 \mathrm{~m}$ para el eje menor. Se realizaron del mismo modo variaciones en el número de vecinos que han fluctuado entre 5 y 3 y entre 9 y 7 , mientras que el valor de $\mathrm{p}$ ha variado entre 1 y 3. Para determinar la orientación de la elipse se probaron valores que oscilan entre $30^{\circ}$ y $45^{\circ}$. 


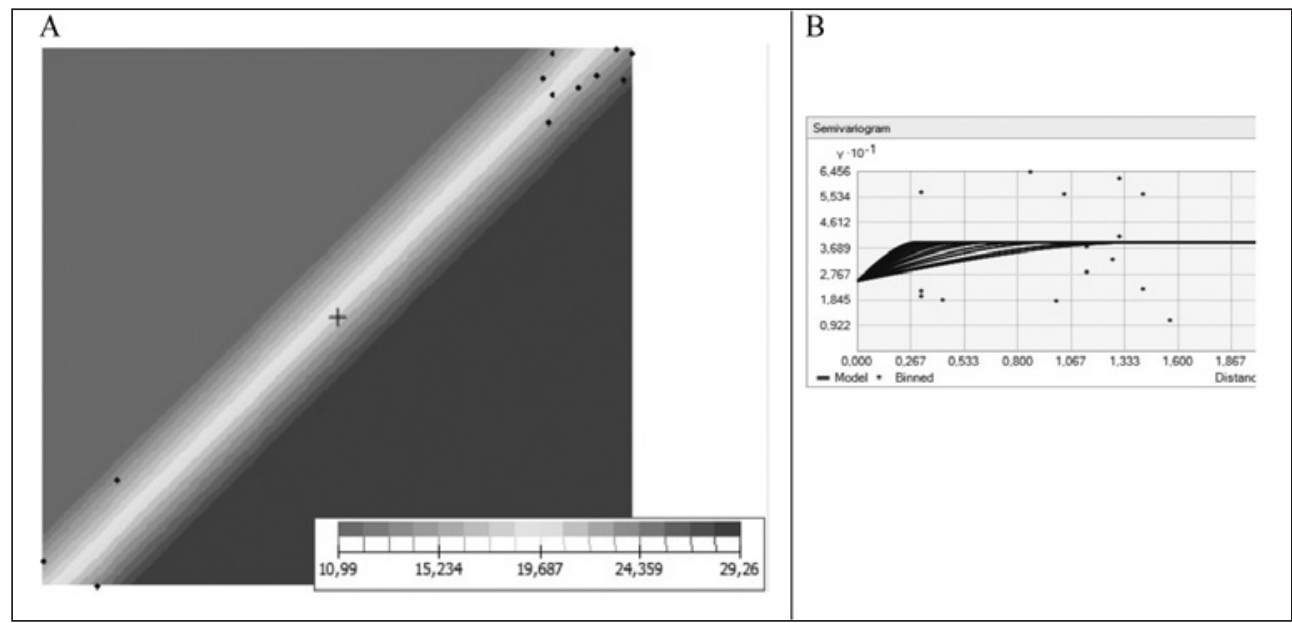

Fuente: Elaboración propia.

\subsubsection{Interpolación espacial de la polución por $\mathrm{NO}_{2}$ y comparación de soluciones}

\section{a) Aplicación del Kriging y del IDW}

Inicialmente el método Kriging isotrópico fue aplicado con los parámetros por defecto, si bien su resultado fue desechado por no ajustarse a la realidad geográfica del municipio. Posteriormente se realizaron distintas pruebas modificando el diámetro del círculo, el número de vecinos, así como el número y amplitud de los intervalos hasta conseguir un modelo más acorde con la trama urbana.

El método Kriging con anisotropía mostró mejores resultados, tanto en la validación del modelo (tabla 8), como en su configuración espacial, sin embargo suaviza mucho los valores pronosticados, aumentando la diferencia entre los valores observados y estimados (tabla 10).

Los resultados obtenidos con la técnica IDW reflejan errores de predicción (RMSE) más bajos con el modelo anisotrópico que con el isotrópico; además, como se verá más adelante, este modelo se ajusta mejor al contexto geográfico. Sin embargo, los resultados logrados con esta técnica presentan unos errores cuadráticos medios ligeramente superiores a los obtenidos con la técnica Kriging (tabla 9).

De la comparación de los estadísticos descriptivos (tabla 10) de ambos modelos se extrae que la media de los datos calculados por todos los modelos es bastante parecida y próxima al valor medio observado. Donde surgen mayores divergencias es en los valores máximos y mínimos estimados. Mientras que el IDW produce un mejor ajuste entre los valores observados y calculados, el Kriging recorta los valores máximos y aumenta los valores mínimos, produciendo un mayor suavizado. En ambas técnicas, los resultados obtenidos con el modelo anisotrópico fueron mejores que con el isotrópico. 
Tabla 8

PARÁMETROS UTILIZADOS Y BONDAD DE AJUSTE CON EL MÉTODO DE INTERPOLACIÓN KRIGING (SANTA CRUZ DE TENERIFE NO 2012)

\begin{tabular}{|l|c|c|c|c|c|c|c|}
\hline TÉCNICA & \multicolumn{3}{|c|}{ PARAMETROS DEL MODELO } & \multicolumn{3}{|c|}{ VALIDACIÓN CRUZADA } \\
\hline & $\begin{array}{c}\text { Norma / } \\
\text { vecinos } \\
\text { sectores/ } \\
\text { rotación/ } \\
\text { tamaño/ } \mathrm{m}\end{array}$ & Ángulo & $\begin{array}{c}\text { Factor de } \\
\text { anisotropía }\end{array}$ & $\begin{array}{c}\bar{X} \\
\text { errores }\end{array}$ & RMSE & RMSSE \\
\hline $\begin{array}{l}\text { KRIGING } \\
\text { ISOTROPICO }\end{array}$ & $8 / 6$ & $\begin{array}{l}\text { Círculo, 4 } \\
\text { partes, 45 } \\
1657\end{array}$ & 0 & 1 & $-0,04$ & 6,09 & 1,06 \\
\hline $\begin{array}{l}\text { KRIGING } \\
\text { ANISOTRÓPICO }\end{array}$ & $7 / 3$ & $\begin{array}{l}\text { Círculo, } 4 \\
\text { partes, } 45^{\circ}, \\
15000 / 3000\end{array}$ & 43 & 5 & 0 & 5,92 & 1,01 \\
\hline
\end{tabular}

Fuente: Elaboración propia.

Tabla 9

PARÁMETROS UTILIZADOS Y BONDAD DE AJUSTE CON EL MÉTODO DE INTERPOLACIÓN IDW (SANTA CRUZ DE TENERIFE NO 2012)

\begin{tabular}{|c|c|c|c|c|c|c|c|}
\hline \multirow[t]{2}{*}{ TÉCNICA } & \multicolumn{5}{|c|}{ PARAMETROS DEL MODELO } & \multicolumn{2}{|c|}{$\begin{array}{c}\text { VALIDACIÓN } \\
\text { CRUZADA }\end{array}$} \\
\hline & $\mathrm{p}$ & $\begin{array}{c}\mathrm{N}^{\mathrm{o}} \\
\text { vecinos }\end{array}$ & $\begin{array}{l}\text { Forma / } \\
\text { sectores / } \\
\text { rotación / } \\
\text { tamaño m }\end{array}$ & Ángulo & $\begin{array}{c}\text { Factor } \\
\text { anisotropía }\end{array}$ & $\begin{array}{c}\bar{X} \\
\text { errores }\end{array}$ & RMSE \\
\hline $\begin{array}{l}\text { IDW } \\
\text { ISOTRÓPICO }\end{array}$ & 1 & $9 / 7$ & $\begin{array}{c}\text { Círculo, } 4 \\
\text { partes, } 45^{\circ} \text {, } \\
3500\end{array}$ & 0 & 1 & 0,04 & 6,6 \\
\hline $\begin{array}{l}\text { IDW } \\
\text { ANISOTRÓPICO }\end{array}$ & 1 & $5 / 3$ & $\begin{array}{c}\text { Círculo, } 4 \\
\text { partes, } 0^{\circ} \text {, } \\
10300 / 2500\end{array}$ & 42 & 4,12 & $-0,11$ & 6,09 \\
\hline
\end{tabular}

Fuente: Elaboración propia.

b) Comparación de los patrones espaciales de $\mathrm{NO}_{2}$ interpolados

- Patrones espaciales de la polución generados con Kriging isotrópico y anisotrópico

Desde el punto de vista de la calidad del aire cabe destacar que la ciudad de Santa Cruz de Tenerife cumple con la legislación española, comunitaria y con la de la Organización Mundial de la Salud, ya que incluso sus valores máximos se encuentran por debajo del nivel medio admisible establecido por dichas organizaciones $\left(40 \mu \mathrm{g} / \mathrm{m}^{3}\right)$. 
Tabla 10

COMPARACIÓN DE LOS ESTADÍSTICOS DESCRIPTIVOS DE AMBOS MÉTODOS Y MODELOS, SANTA CRUZ DE TENERIFE 2012

\begin{tabular}{|l|c|c|c|c|c|}
\hline \multirow{2}{*}{$\begin{array}{l}\text { ESTADÍSTICOS } \\
\text { DESCRIPTIVOS }\end{array}$} & \multirow{2}{*}{$\begin{array}{c}\text { DATOS } \\
\text { OBSERVADOS }\end{array}$} & IDW & IDW & KRIGING & KRINGING \\
\cline { 3 - 6 } & & ISOTRÓPICO & ANISOTRÓPICO & ISOTRÓPICO & ANISOTRÓPICO \\
\cline { 3 - 6 } & 20,52 & 20,94 & 20,35 & 20,24 & 20,35 \\
\hline Media & 5,51 & 0,85 & 1,99 & 0,99 & 1,65 \\
\hline Desviación típica & 29,26 & 28,12 & 28,28 & 23,33 & 24,34 \\
\hline Máximo & 10,99 & 16,15 & 15,89 & 18,13 & 14,92 \\
\hline Mínimo & 18,27 & 11,97 & 12,39 & 5,2 & 9,42 \\
\hline Amplitud & & & & & \\
\hline
\end{tabular}

Fuente: Elaboración propia.

Los patrones obtenidos a partir del Kriging isotrópico (figura 8A) y anisotrópico (figura 8B), presentan una configuración espacial bastante diferente. El patrón obtenido con el modelo isotrópico resulta del todo incoherente con la estructura urbana, ya que presenta cambios bruscos entre píxeles muy próximos, así como geometrías poco plausibles. Estas cuestiones están en estrecha relación con el escaso número de datos observados y la mala distribución de los mismos.

En ambos casos, la superficie representada por el primer intervalo, inferior a $20 \mu \mathrm{g} / \mathrm{m}^{3}$, ocupa la mayor parte del territorio, el 46,85 \% (972,75 ha) en el modelo isotrópico y el 46,69 \% (969,5 ha) en el modelo anisotrópico (tabla 11).

Figura 8

PATRONES ESPACIALES DE LA CONTAMINACIÓN POR NO 2 MEDIANTE EL MÉTODO KRIGING ISOTRÓPICO (A) Y ANISOTRÓPICO (B) EN SANTA CRUZ DE TENERIFE 2012

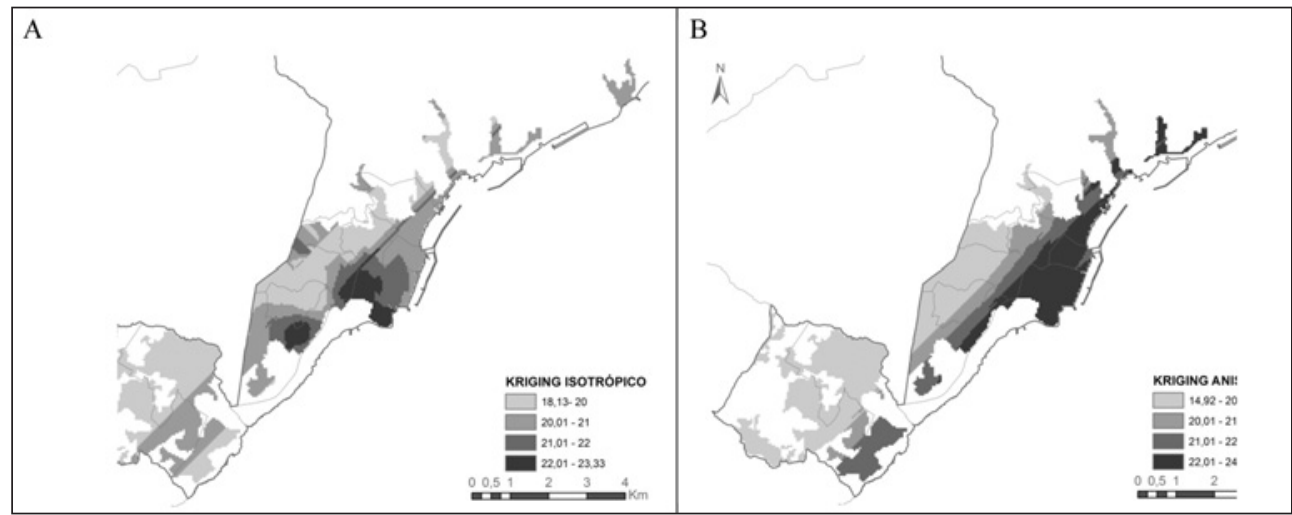

La configuración espacial generada por el modelo anisotrópico resulta bastante compatible con el contexto geográfico local, además de presentar cambios graduales entre los 
intervalos y una convincente orientación general NE-SW, como se comprobó al calcular la anisotropía. Su bondad de ajuste, tal y como se observó antes, es ligeramente superior a la del método IDW anisotrópico. Su principal inconveniente es que presenta un mayor suavizado.

Tabla 11

SUPERFICIE OCUPADA POR LOS DIFERENTES NIVELES DE NO 2 CON EL MÉTODO KRIGING, SANTA CRUZ DE TENERIFE 2012

\begin{tabular}{|c|c|c|c|c|}
\hline $\begin{array}{c}\text { INTERVALOS } \\
\mu \mathrm{g} / \mathrm{m}^{3}\end{array}$ & \multicolumn{2}{|c|}{$\begin{array}{c}\text { KRIGING } \\
\text { ISOTRÓPICO }\end{array}$} & \multicolumn{2}{c|}{$\begin{array}{c}\text { KRIGING } \\
\text { ANISOTRÓPICO }\end{array}$} \\
\hline & Superficie ha & $\%$ & Superficie ha & $\%$ \\
\hline$<20$ & 972,75 & 46,85 & 969,5 & 46,69 \\
\hline $20-21$ & 739 & 35,59 & 294 & 14,16 \\
\hline $21-22$ & 226 & 10,89 & 354,25 & 17,06 \\
\hline$>22$ & 138,5 & 6,67 & 458,5 & 22,08 \\
\hline
\end{tabular}

- Patrones espaciales de la polución generados con IDW isotrópico y anisotrópico

La configuración espacial obtenida con el modelo isotrópico (figura 9A) vuelve a mostrar, como en Barcelona, los conocidos «ojos de buey», con los tonos más oscuros en torno a los valores más bajos y los colores más claros en torno a los valores más altos. Por otra parte, este modelo presenta una gran concentración de la superficie ocupada en torno a los intervalos centrales 19 y $22 \mu \mathrm{g} / \mathrm{m}^{3}$, con cerca de $88 \%$ del área urbana poblada (tabla 12).

Tabla 12

ÁREA OCUPADA POR LOS DISTINTOS NIVELES DE NO INTERPOLADOS CON EL MÉTODO IDW

\begin{tabular}{|l|c|c|c|c|}
\hline $\begin{array}{c}\text { INTERVALOS } \\
\mu \mathrm{g} / \mathrm{m}^{3}\end{array}$ & \multicolumn{2}{|c|}{ IDW } & \multicolumn{2}{c|}{ IDW } \\
& ISOTRÓPICO & $\%$ & Superficie ha & $\%$ \\
\hline$<19$ & 14 & 0,67 & 777,75 & 37,46 \\
\hline $19-20,5$ & 704,25 & 33,92 & 405,75 & 19,54 \\
\hline $20,5-22$ & 1118 & 53,85 & 282,25 & 13,59 \\
\hline$>22$ & 240 & 11,56 & 610,5 & 29,40 \\
\hline
\end{tabular}

La configuración espacial generada por el IDW anisotrópico (figura 9B) se parece bastante a la del Kriging anisotrópico, si bien la primera muestra una configuración menos suavizada. La superficie ocupada por los diferentes intervalos está repartida de modo más homogéneo que en el Kriging (tabla 12). En ambos casos los valores de $\mathrm{NO}_{2}$ disminuyen desde la costa hacia el NW, a medida que nos alejamos del centro de la ciudad y la altitud es mayor. 


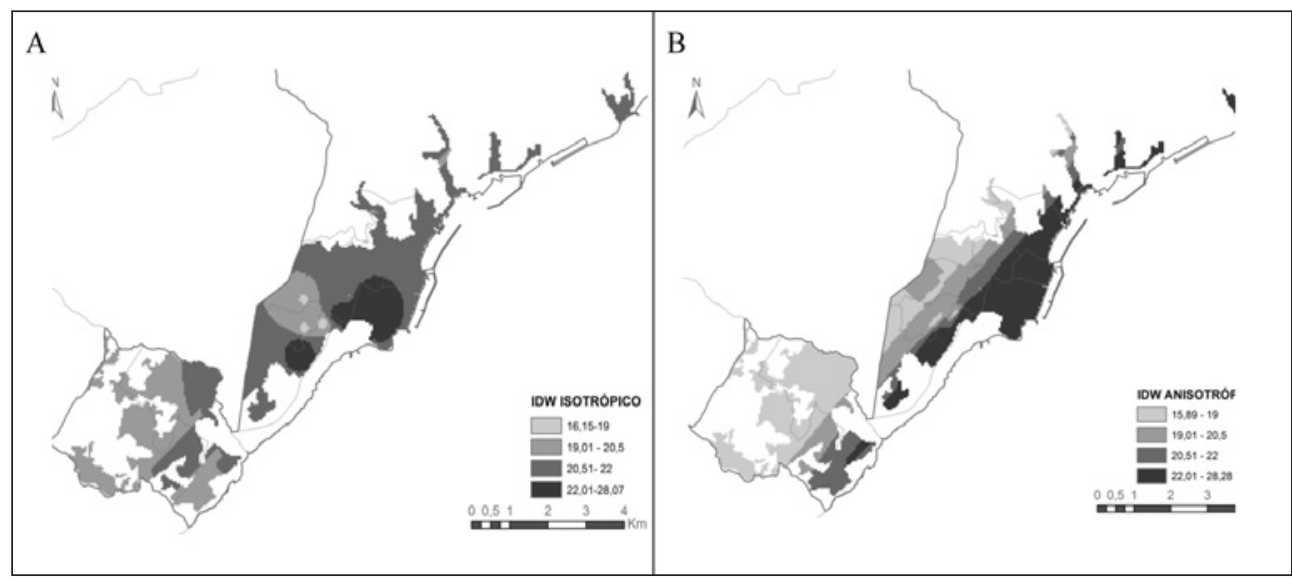

La superficie generada con el IDW anisotrópico, sin embargo, presenta algunas incoherencias espaciales ligeramente mayores que la del Kriging anisotrópico, que unidas a unos valores de RMSE más altos, hacen que sea difícil anteponerla a la del Kriging en la ciudad de Santa Cruz de Tenerife. Por su parte, en contra del Kriging está el mayor recorte que se produce en los valores estimados máximos y mínimos frente a los observados, cuando la capa geosestadística resultante de la interpolación se exporta a raster y se ajusta a la zona urbana poblada. En suma, los criterios no avalan una elección clara.

\section{CONCLUSIONES}

En este trabajo se ha abordado un tema crucial en los estudios ambientales como es la estimación de valores de contaminación en lugares donde no existen mediciones, a partir de una muestra de observaciones empíricas realizadas sobre una serie de puntos o estaciones, aplicando técnicas de interpolación espacial. En él se ha ensayado un protocolo de actuación que ayude a tomar de decisiones técnicas para mejorar dichas estimaciones sobre la contaminación urbana con datos insuficientes y a costes asequibles.

Metodológicamente el trabajo se ha desarrollado en tres etapas, cada una de ellas aportando utilidades diferenciadas hacia esa meta general. En la primera se ha explorado la distribución univariada de los datos muestrales con varias herramientas y se ha investigado la existencia de comportamientos anisotrópicos en la distribución espacial del $\mathrm{NO}_{2}$ mediante el semivariograma, supuesto que se ha corroborado.

En la segunda etapa se ha realizado el análisis estructural de los datos muestrales, recurriendo a dos técnicas complementarias: por un lado el ajuste de una función sobre el semivariograma empírico, para calcular de modo objetivo los parámetros de la anisotropía espacial de la contaminación por $\mathrm{NO}_{2}$. En concreto los que han logrado unos resultados mejores para Barcelona han sido: unas dimensiones de la elipse de $9000 \mathrm{~m}$ por $5000 \mathrm{~m}$, 
una orientación de $30^{\circ}$, un número de vecinos entre 6 y 4 y la elipse dividida en cuatro partes de $45^{\circ}$. Para la ciudad de Tenerife los mejores parámetros han sido $15000 \mathrm{~m}$ y 3000 $\mathrm{m}$ para los ejes de la elipse, la orientación de $43^{\circ}$, vecinos entre 7 y 4 y la elipse, como en Barcelona dividida en 4 partes de $45^{\circ}$. A ello se ha añadido el uso del GPI (o análisis de superficie de tendencia) para desvelar la tendencia general latente en la distribución espacial del contaminante. Ambos resultados han tenido un gran peso en las decisiones metodológicas adoptadas en la fase siguiente.

En la tercera y última etapa se han aplicado dos técnicas de interpolación: la IDW y el Kriging ordinario. Con los estadísticos obtenidos se han podido validar los modelos resultantes y comparar los patrones espaciales de la contaminación por $\mathrm{NO}_{2}$.

Los patrones espaciales generados presentan diferencias notables entre ellos, si bien los más semejantes son los del IDW y Kriging anisotrópicos. A la hora de elegir entre ellos, y sobre la base de criterios estadísticos y cualitativos, resultaría preferible la solución del IDW anisotrópico para la ciudad de Barcelona y, aunque menos clara, la del Kriging anisotrópico, para Santa Cruz de Tenerife. Ambas soluciones se han obtenido mediante ensayos sistemáticos, en las cuales se aúnan unos indicadores de bondad de ajuste aceptables (pero no necesariamente los mejores) y unos valores estimados más coherentes con la estructura urbana y el marco territorial.

Como balance cabe subrayar que la dificultad de generar estimaciones de la contaminación para un espacio urbano completo, cuando los datos son escasos y poseen una representatividad territorial limitada, puede verse paliada recurriendo al uso inteligente de una batería de herramientas de análisis y modelado espacial. Con un procedimiento como el aquí adoptado es posible avanzar, sobre bases más sólidas, hacia un conocimiento más completo de los patrones de contaminación local, que permitan luego su inserción en estudios y políticas sobre calidad y justicia ambiental, morbilidad y salud ambiental, malestar socio-ambiental, sostenibilidad, etc.

\section{RECONOCIMIENTOS}

Este trabajo se ha elaborado dentro del proyecto de investigación «Contaminación atmosférica urbana y justicia ambiental: metodología de evaluación y estudio de casos con sistemas de información geográfica», financiado por el Ministerio de Ciencia e Innovación de España, referencia CSO2011-26177.

Se agradece al Dr. Antonio Palacios García los trabajos de delimitación del área urbana poblada de la ciudad de Barcelona.

\section{BIBLIOGRAFÍA}

BABAK, O. y DEUTSCH, C. (2009): «Statistical approach to inverse distance interpolation», Stoch. Environ. Res. and Risk Assess., 23, pp. 543-553.

BALDASANO, J. M., GÜERECA, L.P., LÓPEZ, E., GASSOA, S. Y JIMENEZ-GUERRERO, P. (2008): «Development of a high-resolution (1 km $\times 1 \mathrm{~km}, 1 \mathrm{~h})$ emission model for Spain: The High-Elective Resolution Modelling Emission System (HERMES)», Atmospheric Environment, 42 (31), pp. 7215-7233. 
CAÑADA TORRECILLA, R. (2007): «Técnicas de interpolación espacial deterministas y exactas: media ponderada por el inverso de la distancia y funciones de base radial», en MORENO JIMÉNEZ, A. (Coord.): Sistemas y análisis de la información geográfica. Madrid, Ra-Ma, 781-809.

CAÑADA TORRECILLA, R. (2007): «Técnicas de interpolación geoestadísticas: Kriging ordinario», en MORENO JIMÉNEZ, A. (Coord.): Sistemas y análisis de la información geográfica. Madrid, Ra-Ma, pp. 823-853.

CAÑADA TORRECILLA, R. (2012): «Cambios espaciales y temporales en la contaminación por dióxido de nitrógeno en el municipio de Madrid (2001-2011)», Nimbus, 29-30, pp. 127-143.

CAÑADA TORRECILLA, R., VIDAL DOMÍNGUEZ, M. J. y MORENO JIMÉNEZ, A. (2011): «Interpolación espacial y visualización cartográfica para el análisis de la justicia ambiental: ensayo metodológico sobre la contaminación por partículas atmosféricas en Madrid», GeoFocus (Artículos), 11, p. 118-154. Disponible en http://geofocus.rediris. es/2011/Articulo6_2011.pdf

DALY, A. y P. ZANNETTI (2007): «Air pollution modeling - An overview», en P. ZANNETTI, D. AL-AJMI, y S. AL-RASHIED (Eds.), Ambient air pollution, Chapter 2. The Arab School for Science and Technology (ASST) and The Enviro. Comp. Institute.

DE MESNARD, L. (2013): «Pollution models and inverse distance weighting: some critical remarks», Computers and Geosciences, 52, pp. 459-469.http://www.sciencedirect.com/ science/article/pii/S009830041200372X

DIEM, J.E. y COMRIE, A.C. (2002): «Predictive mapping of air pollution involving sparse spatial observations», Environmental Pollution, 119, pp. 99-117.

EEA (2012): Air quality in Europe. Report $n^{o}$ 4, Luxembourg, 104 p. http://ec.europa.eu/ environment/air/pollutants/ceilings.htm

HENGL, T. (2011): A practical guide to geostatistical mapping of environmental variables. Amsterdam, University of Amsterdam, $291 \mathrm{pp}$.

KRIVORUCHKO, K. 2011. Spatial Statistical Data Analysis for GIS Users (DVD-ROM), Esri Press.

LI, J. y HEAP, A.D. (2008): A review of spatial interpolation methods for environmental scientists. Camberra, Geoscience Australia, 137 p.

LI, J. y HEAP, A.D. (2010): «A review of comparative studies of spatial interpolation methods in environmental sciences: Performance and impact factors», Ecological Informatics, 6, pp. 228-241.

MONMONIER, M. (1997): Cartographies of danger: mapping hazards in America. Chicago, The University of Chicago Press.

MORENO JIMÉNEZ, A. (2013): «Población y polución atmosférica intraurbana por dióxido de nitrógeno en Madrid: análisis desde la justicia ambiental basado en sistemas de información geográfica», Cuadernos Geográficos de la Universidad de Granada (en prensa).

MORENO JIMÉNEZ, A. y CAÑADA TORRECILLA, R. (2007): «Justicia ambiental y contaminación atmosférica por dióxido de azufre en Madrid: análisis espacio-temporal y valoración con sistemas de información geográfica», Boletín de la Asociación de Geógrafos Españoles, 44, pp. 301-324. http://www.boletinage.com/44/14-moreno.pdf 
MUELLER, T.G., PUSULURI, N.B., MATHIAS, K.K., CORNELIUS, P.L., BARNHISEL, R.I, SHEARER, S.A. (2004): «Map quality for ordinary Kriging and inverse distance weighted interpolation», Soil Science Society of America Journal, 68, pp. 2042-2047.

WILLIAM R., GREGORY, M.J. y SEINFELD, J.H. (1979): «A comparison of interpolation methods for sparse data: application to wind and concentration fields», Journal of Applied Meteorology, 18 (6), pp. 761-771.

WONG, D., YUAN, L. y PERLIN, S.A. (2004): «Comparison of spatial interpolation methods for the estimation of air quality data», Journal of Exposure Analysis and Environmental Epidemiology 14(5), pp. 404-415.

PARRA, R. (2004): Development of the EMICAT2000 model for the estimation of air pollutants emissions from Catalonia and its use in photochemical dispersion modelling. Ph.D. Thesis. Technical University of Catalonia. 